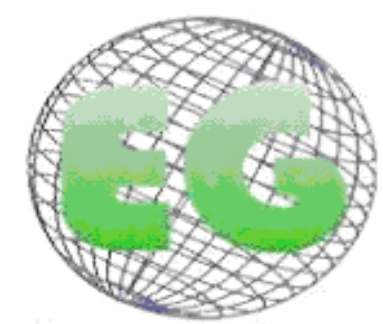

ISSN 1695-6141

N24

\title{
Estudio comparativo de la presión arterial invasiva frente a la presión arterial no invasiva. Valoración de la diferencia.
}

Comparative study of invasive blood pressure versus non-invasive blood pressure

\author{
"Simarro Blasco, JA., "Noheda Blasco, MC., "**Bascuñana Blasco, M., \\ ${ }^{* * * *}$ Noheda Recuenco, M., ${ }^{* * * * *}$ Tolmo Aranda, I., "*****Romero Carralero, MI. \\ *Enfermeros UCI Hospital Virgen de la Luz de Cuenca - SESCAM.
}

\begin{abstract}
Palabras clave: Cuidados intensivos; Determinación de la presión sanguínea; Efecto de drogas; Enfermería; Presión arterial; Respiración artificial; Presión sanguínea; Presión sistólica y Presión diastólica.
\end{abstract}

Keywords: Intensive care; determination of blood pressure: effect of drugs; nursing; blood pressure; artificial respiration; systolic pressure and diastolic pressure.

\section{RESUMEN}

En la práctica clínica, la tensión arterial es un parámetro importante en la valoración hemodinámica del paciente crítico. Existen dos formas de medición: Presión Invasiva (PI) y Presión No Invasiva (PNI). Como objetivos nos planteamos comparar la diferencia entre ambas formas de medición, valorar las posibles diferencias por la localización arterial, edad y sexo, valorar la influencia de la ventilación mecánica, drogas inotrópicas y antecedentes personales en la diferencia de Presión arterial Invasiva (PI) respecto a la Presión arterial No Invasiva (PNI). Identificar su significación clínica y estimar la fiabilidad de la PNI. Hicimos un estudio descriptivo, longitudinal y comparativo de marzo a diciembre 2008. Se analizó sexo, edad y localización arterial (hemicuerpo y arteria). Incluimos pacientes con arteria con curva óptima y calibración en el momento de medición en decúbito supino. Se tomó la PNI en ambos brazos con 5 minutos de diferencia, registrando la PI al mismo tiempo. Análisis con el Spss 15.0. Se analizó la diferencia en 109 pacientes con diferencias estadísticamente significativas en algún parámetro analizado. Como conclusiones y discusión planteamos que las diferencias encontradas no son clínicamente significativas y por tanto la PI y la PNI son fiables en estado óptimo de calibración.

\section{ABSTRACT}

In clinical practice, blood pressure is an important parameter in patient critic hemodynamic valuation. There are two ways of measuring: Invasive Pressure (IP) and Non Invasive Pressure (NIP). As objectives, we set ourselves the task of comparing the difference between both forms of measurement, assessing the possible differences by arterial location, age and sex, assessing the influence of mechanical ventilation, inotropic drugs and casework in invasive blood pressure difference (IP) and non invasive arterial pressure (NIP). We sought to identify its clinical significance and estimate the reliability of the NIP. We conducted a descriptive, longitudinal and comparative study between March and December 2008. Sex, age and arterial location (hemi-body and 
artery) were analyzed. We have included patients with artery with optimal curve and calibration at the time of measurement in decubitus. NIP was taken in both arms at an interval of five minutes, and IP was recorded at the same time. Analysis was with SPSS 15.0. The difference was analyzed in 109 patients with statistically significant differences in any scanned parameters. Under discussion and conclusions we propose that the differences found are not clinically significant and therefore the IP and the NIP are reliable in optimal calibration state.

\section{INTRODUCCIÓN}

En la Unidad de Cuidados Intensivos (UCl) ingresan normalmente pacientes con gran inestabilidad hemodinámica en los que puede haber fallo de un órgano o fallo multiorgánico. Son pacientes que requieren estancias muy prolongadas. Estas circunstancias hacen preciso un control exhaustivo de los parámetros vitales más importantes, entre ellos la presión arterial (PA). Debemos tener valores más o menos continuos en función de las características del paciente y su estabilidad. De forma rutinaria en las UCls existen 2 formas de tomar la PA: PA invasiva a través de canalizar una arteria, o PA no invasiva a través de manguito tradicional, que en la mayoría de las unidades está conectado a la monitorización del paciente. ${ }^{(1)(2)}$

La canalización arterial con propósitos clínicos fue descrita por primera vez a principios de los años 50 y es en la actualidad una de las técnicas más comunes en las Unidades de Cuidados Intensivos. Esta técnica se utiliza con dos propósitos principales, por un lado el control de la PA de forma continua y por otro sacar analíticas de forma frecuente sin necesidad de repetidas punciones. Tiene una fiabilidad contrastada y una exactitud acreditada si la trasmisión de curva de presiones es correcta. ${ }^{(3)}$

Por otro lado, la forma de medir la PA con manguito de presiones de forma no invasiva está avalada por estudios. Los técnicos de mantenimiento revisan de forma usual estos aparatos, dando fiabilidad y exactitud a la medición. ${ }^{(4)(5)}$

Está acreditada la diferencia de tensión arterial entre diferentes arterias, aunque de forma usual esta diferencia es mínima entre miembros superiores, y si es mayor de $10 \mathrm{~mm}$ de $\mathrm{Hg}$ habría que investigar patologías asociadas. ${ }^{(6)(7)(8)}$

Hay patologías de base en los pacientes que afectan a la PA. Sabemos que la HTA, DM, dislipemias, arteriopatías, fumadores y obesidad puede alterar la contractibilidad y a su vez la PA. ${ }^{(7)(8)}$

La ventilación mecánica afecta al retorno venoso al introducir aire con presión al tórax, y en parámetros, como la peep, deja una presión residual alterando la PA. ${ }^{(9)}$

Medicamentos usados de forma frecuente en $\mathrm{UCl}$, como la norepinefrina y la dopamina, van pautados para controlar la estabilidad hemodinámica y por lo tanto mejorar la PA. ${ }^{(9)(10)}$

En la práctica diaria de enfermería, surgen problemas de credibilidad en la medición tanto de forma invasiva como de forma no invasiva en la PA.

Tras varios problemas de este tipo nos planteamos como objetivo realizar un estudio para comparar ambos métodos de medición de presión arterial en el mismo paciente al mismo tiempo y determinar así la fiabilidad de PA invasiva y PA no invasiva. El equipo investigador decidió valorar la presencia o ausencia de todos los factores expuestos anteriormente y acreditados en la bibliografía para comprobar si por sí solos o asociados entre sí afectan a la 
PA invasiva respecto a la PA no invasiva, es decir, la diferencia entre PA invasiva-PA no invasiva.

\section{MATERIAL Y MÉTODO}

Planificamos un estudio descriptivo, longitudinal y comparativo desde marzo a diciembre de 2008.

El equipo investigador se aseguró que los equipos electrónicos, tanto de PA invasiva como de PA no invasiva estaban calibrados por técnicos y funcionaban correctamente.

Incluimos a todos los pacientes con arteria canalizada y con el criterio de curva de PA, de dos miembros del equipo investigador, correcta. Si al paciente se le cambiaba la localización de la arteria canalizada se la hacía otra muestra de medición.

Se excluyeron a los pacientes que no podían permanecer en decúbito supino durante todo el protocolo de medición o había potencial riesgo en la medición.

El protocolo para hacer la medición se decidió por el equipo investigador:

El paciente se colocaba en decúbito supino al menos 5 minutos, se hacía una calibración y asegurábamos que el transductor de presiones estaba colocado a la altura de la aurícula derecha, y se hacía una medición de PA no invasiva en un miembro superior, anotando a la vez la PA invasiva detectada. A los 5 minutos se hacía una nueva medición en el miembro superior opuesto con PA no invasiva y se anotaba también la PA invasiva de este momento. Registramos presencia o ausencia de:

- Ventilación Mecánica

- Antecedentes personales diagnosticados en la historia médica (Diabetes Mellitus, HiperTensión Arterial, Fumador, Arteriopatía y dislipemias)

- Drogas inotrópica (Dopamina o Norepinefrina).

Se hizo un análisis de la arteria canalizada y en el hemicuerpo que estaba localizada (Derecho o Izquierdo).

Por último registramos variables socio-demográficas: edad y sexo. El análisis estadístico se ha realizado con el programa SPSS 15.0. Se ha utilizado la prueba ANOVA, el test de Kruskal-Wallis y $\mathrm{T}$ de Student, con una significación estadística $\mathrm{p}<0.05$ y un intervalo de confianza del $95 \%$.

\section{RESULTADOS}

Se analizó la Presión Arterial a 109 pacientes con una edad media de 62.66 años y una desviación estándar de 17.14 años.

El $73.4 \%(n=80)$ fueron hombres y el $26.6 \%(n=26)$ mujeres. 
Describiendo los antecedentes personales analizados, el $37.6 \%$ de los pacientes tenían Hiper-Tensión Arterial (HTA), el 32.1\% Diabetes Mellitus (DM), el 35.8\% eran fumadores, $12.8 \%$ sufrían dislipemias y el $2.8 \%$ dislipemias.

Las drogas inotrópicas analizadas, que se utilizaron para la estabilidad hemodinámica de los pacientes analizados, el $44 \%$ estaban con perfusión de Dopamina, el $25.7 \%$ con perfusión de Norepinefrina, con ambas drogas estaba el $21.1 \%$ y ninguna el $51.4 \%$ de los pacientes analizados.

El $61.1 \%$ de los pacientes incluidos en el estudio estaban con ventilación mecánica.

Localización de la Arteria Canalizada:

Arteria radial $81.7 \%$ - Arteria Femoral 14.7\% - Arteria Humeral 1.8\% - Arteria Pedia 1.8\%. Hemicuerpo derecho $55 \%$ frente Hemicuerpo izquierdo $45 \%$.

Arteria radial derecha $43.1 \%$ - Arteria radial izquierda $38.5 \%$ - Femoral derecha $9.2 \%$ Femoral izquierda $5.5 \%$ - Ambas Humerales y Pedias $0.9 \%$ cada una.

La diferencia en PA SISTÓLICA (PAS) entre PA Invasiva y PA No Invasiva encontrada es de $8.71 \pm 10.21 \mathrm{~mm}$ de Hg., como se puede ver en el Gráfico 1

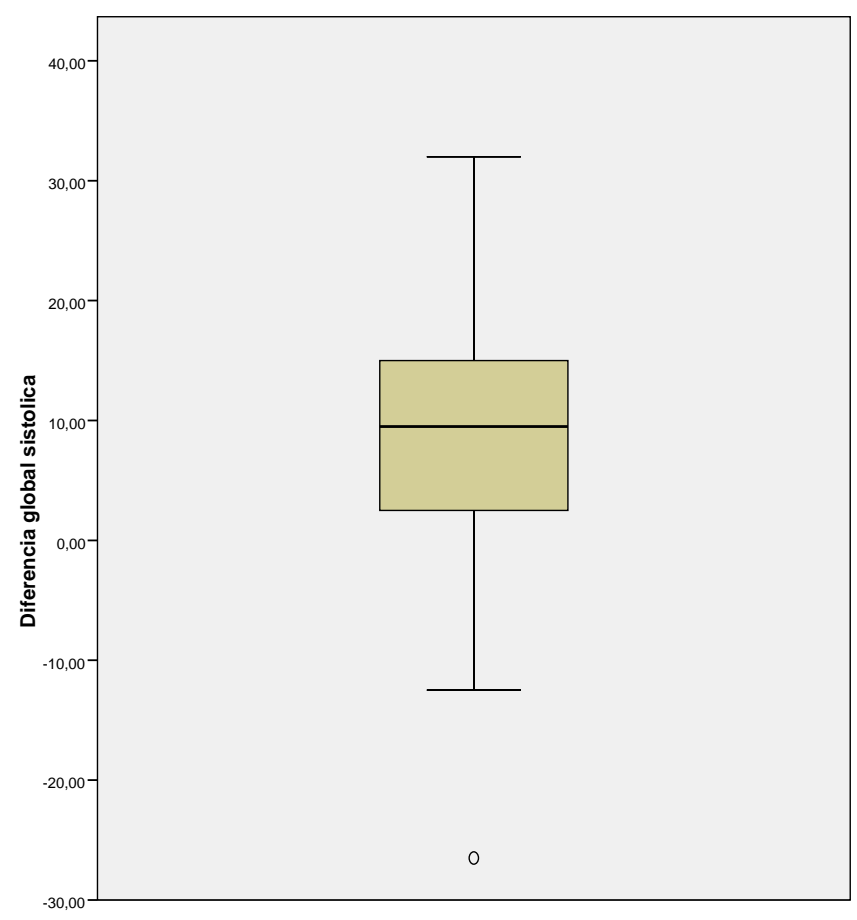

La diferencia en PA DIASTÓLICA (PAD) entre PA Invasiva y PA No Invasiva encontrada es de $5.62 \pm 6.15 \mathrm{~mm}$ de Hg., como se puede ver en el Gráfico 2. 


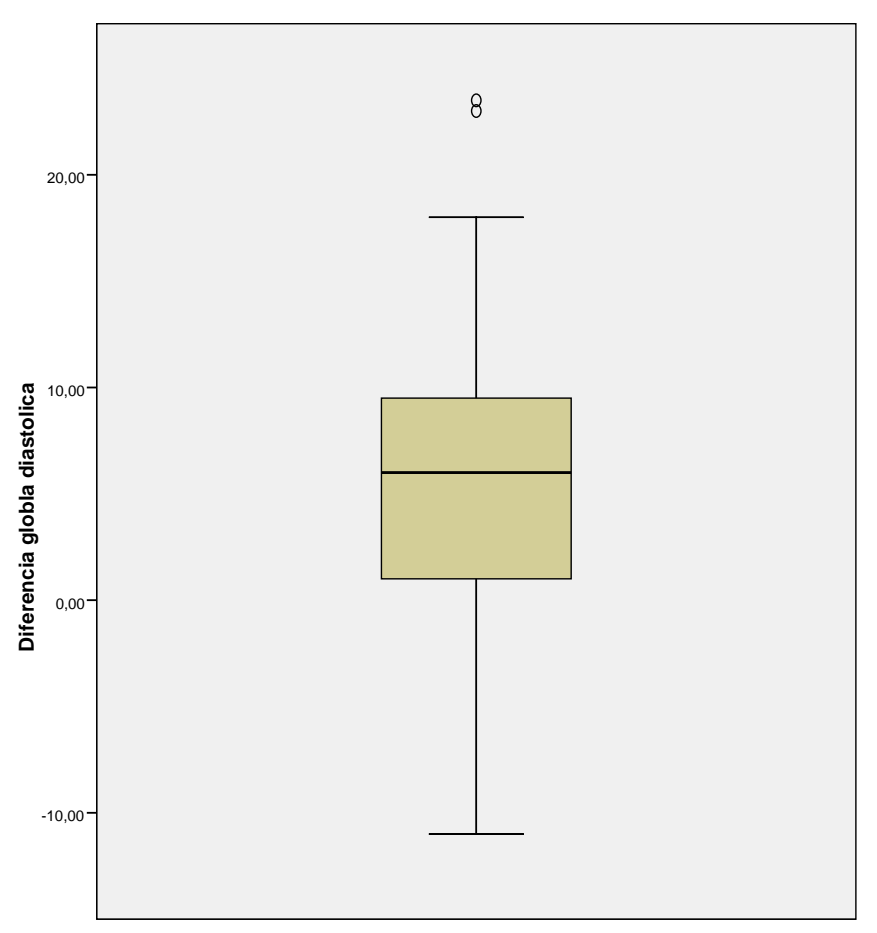

Desglosando las diferencias encontradas en ambas brazos, describimos:

- Diferencia entre PA Invasiva y PA No Invasiva en Miembro Superior Derecho (MSD): PAS $8.72 \pm 11.10$ y PAD $5.75 \pm 6.57 \mathrm{~mm}$ de $\mathrm{Hg}$.

- Diferencia entre PI y PNI en Miembro Superior Izquierdo (MSI): PAS 8.69 \pm 10.95 y PAD $5.49 \pm 7.58 \mathrm{~mm}$ de $\mathrm{Hg}$.

Analizando por sexos se han encontrado diferencias estadísticamente significativas en las siguientes:

- La diferencia de PA DIASTÓLICA entre PA Invasiva y PA No Invasiva en hombres es de 4.68 y en mujeres de 8.22 con $p=0.007$. Gráfico 3: 


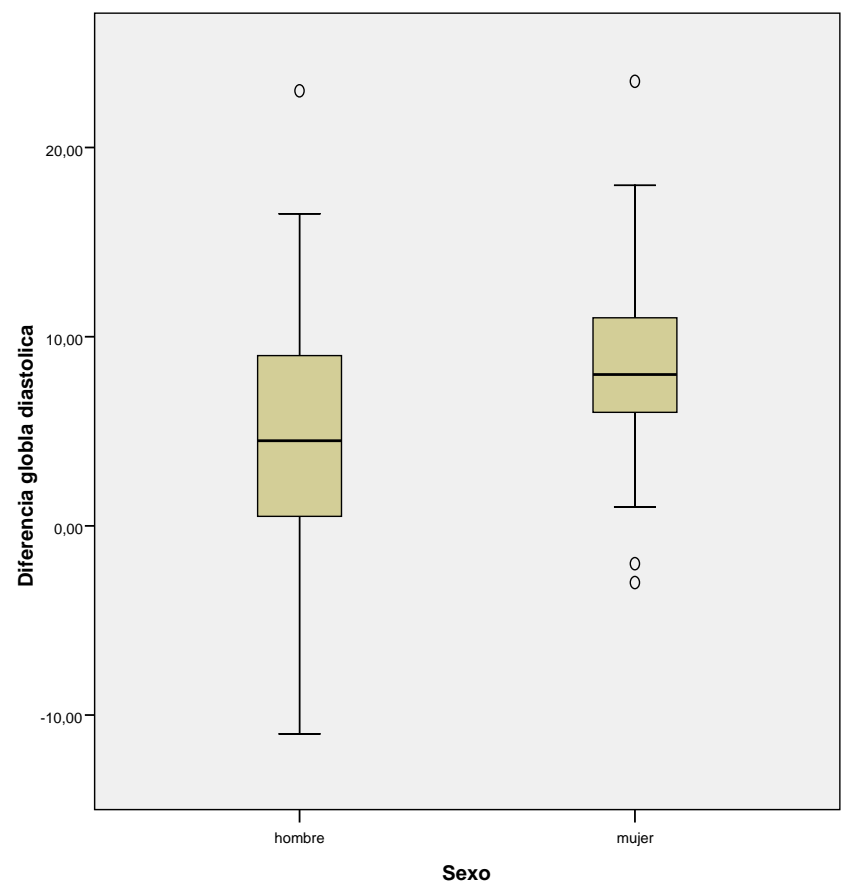

- La diferencia de PA DIASTÓLICA entre PA Invasiva y PA No Invasiva en MSI en hombres es de 4.62 y en mujeres de 7.86 con $p=0.048$. Gráfico 4:

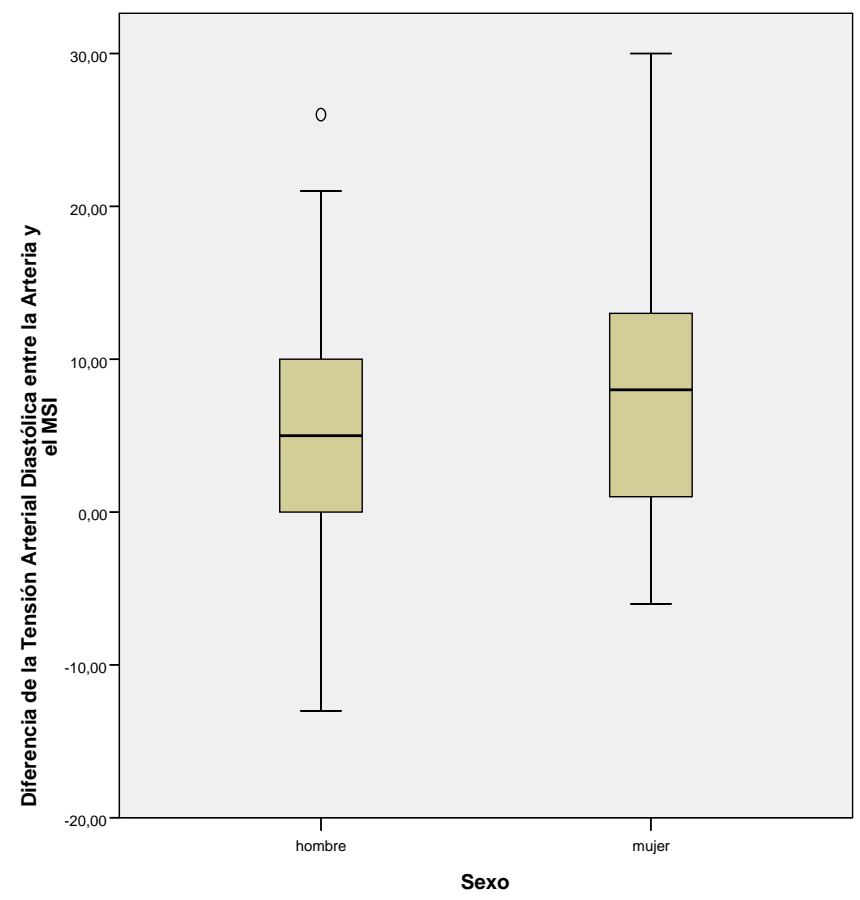

- La diferencia de PA DIASTÓLICA entre PA Invasiva y PA No Invasiva en MSD en hombres es de 4.73 y en mujeres de 8.59 con $p=0.006$. Gráfico 5: 


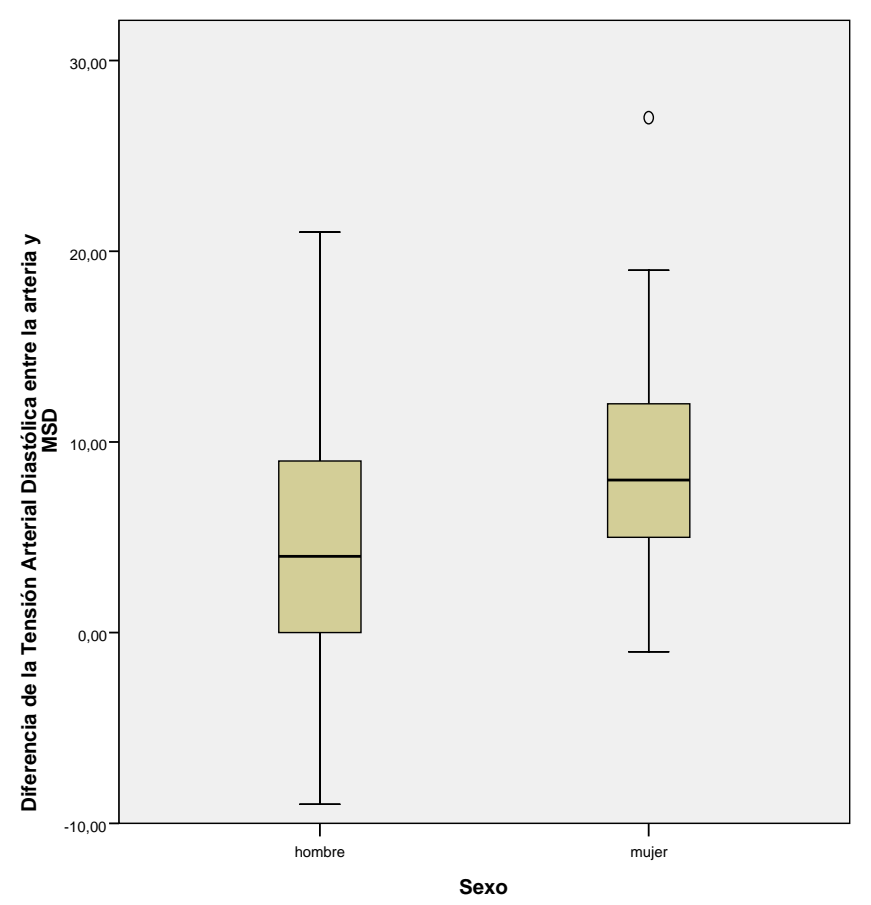

No hay correlación en función de la edad, ni diferencias estadísticamente significativas al analizar los antecedentes personales por individualizado ni al conjugarlos. Tampoco hay diferencias analizando las distintas arterias canalizadas y el hemi-cuerpo de la canalización. La ventilación mecánica tiene efectos estadísticamente significativos en al PA SISTÓLICA como se observa en la siguiente Tabla 1:

\begin{tabular}{|c||c|c|c|}
\hline & $\begin{array}{c}\text { VENTILACIÓN } \\
\text { MECÁNICA } \\
\text { Si }\end{array}$ & $\begin{array}{c}\text { VENTILACIÓN } \\
\text { MECÁNICA } \\
\text { No }\end{array}$ & $p$ \\
\hline \hline $\begin{array}{c}\text { Diferencia } \\
\text { Sistólica }\end{array}$ & $7.12 \mathrm{mmHg}$ & $11.80 \mathrm{mmHg}$ & 0.023 \\
\hline \hline $\begin{array}{c}\text { Diferencia } \\
\text { Diastólica }\end{array}$ & $5.10 \mathrm{mmHg}$ & $6.62 \mathrm{mmHg}$ & 0.224 \\
\hline
\end{tabular}

Tabla 1: Diferencia media de la PA Invasiva con PA No Invasiva en PA sistólica y diastólica respecto a la presencia o ausencia de ventilación mecánica.

Cuando el paciente está con perfusión de drogas inotrópicas nos encontramos los siguientes resultados. Tablas 2, 3 y 4.

\begin{tabular}{|c|c|c|c|}
\hline & $\begin{array}{l}\text { DOPAMINA } \\
\text { Si }\end{array}$ & $\begin{array}{l}\text { DOPAMINA } \\
\text { No }\end{array}$ & $p$ \\
\hline $\begin{array}{l}\text { Diferencia } \\
\text { Sistólica }\end{array}$ & $4.42 \mathrm{mmHg}$ & $12.08 \mathrm{mmHg}$ & $<0.001$ \\
\hline $\begin{array}{l}\text { Diferencia } \\
\text { Diastólica }\end{array}$ & $5.57 \mathrm{mmHg}$ & $5.66 \mathrm{mmHg}$ & 0.945 \\
\hline
\end{tabular}

Tabla 2: Diferencia media de la PA Invasiva con PA No Invasiva en PA sistólica y diastólica respecto a la presencia o ausencia de perfusión de dopamina. 


\begin{tabular}{|l||l||l|l|}
\hline & $\begin{array}{l}\text { NOREPINEFRINA } \\
\text { Si }\end{array}$ & $\begin{array}{l}\text { NOREPINEFRINA } \\
\text { No }\end{array}$ & p \\
\hline \hline $\begin{array}{l}\text { Diferencia } \\
\text { Sistólica }\end{array}$ & $4.54 \mathrm{mmHg}$ & $10.15 \mathrm{mmHg}$ & 0.011 \\
\hline \hline $\begin{array}{l}\text { Diferencia } \\
\text { Diastólica }\end{array}$ & $4.63 \mathrm{mmHg}$ & $5.96 \mathrm{mmHg}$ & 0.323 \\
\hline
\end{tabular}

Tabla 3: Diferencia media de la PA Invasiva con PA No Invasiva en PA sistólica y diastólica respecto a la presencia o ausencia de perfusión de norepinefrina.

\begin{tabular}{|l||l|l|l||}
\hline & $\begin{array}{l}\text { DOPAMINA } \\
\text { NOREPINEFRINA } \\
\text { Si }\end{array}$ & $\begin{array}{l}\text { DOPAMINA Y } \\
\text { NOREPINEFRINA } \\
\text { No }\end{array}$ & $\mathbf{p}$ \\
\hline $\begin{array}{l}\text { Diferencia } \\
\text { Sistólica }\end{array}$ & $2.07 \mathrm{mmHg}$ & $11.74 \mathrm{mmHg}$ & $<0.001$ \\
\hline $\begin{array}{l}\text { Diferencia } \\
\text { Diastólica }\end{array}$ & $4.33 \mathrm{mmHg}$ & $5.63 \mathrm{mmHg}$ & 0.697 \\
\hline
\end{tabular}

Tabla 4: Diferencia media de la PA Invasiva con PA No Invasiva en PA sistólica y diastólica respecto a la presencia o ausencia de perfusión de dopamina y norepinefrina conjuntamente.

\section{CONCLUSIONES Y DISCUSIÓN}

La importancia contrastada de la Presión Arterial en los pacientes en estado crítico, hace imprescindible estar seguros de una medición lo más exacta posible. ${ }^{(1)}$ El trabajo con pacientes en situaciones críticas hace que tengamos muy clara la metodología y que la monitorización, como herramienta de trabajo, sea fiable.

Partimos de la base una canalización correcta y una monitorización adecuada, con una transmisión de onda de presiones buena.

Si estas premisas se cumplen, en el estudio se han encontrado diferencias estadísticamente significativas en la PA Sistólica, cuando sometemos al paciente a terapias propias de la $\mathrm{UCl}$, como Ventilación Mecánica o drogas inotrópicas. También hemos encontrado diferencias significativas en la PA diastólica en función del sexo y el brazo de colocación del manguito

Tras varios análisis interdisciplinarios, concluimos que las diferencias encontradas no crean situaciones clínicas diferentes para $\mathrm{UCl}$, sobre todo en situaciones de emergencia, cuando hay que tomar decisiones rápidas y fiables, para tratamientos inmediatos a corto plazo. Las diferencias son asumibles y por lo tanto hay que fiarse de la monitorización Invasiva o No Invasiva que se tenga en el momento.

Recomendamos una revisión continua de las máquinas y sistemas de monitorización, para evitar suspicacias sobre los valores que aportan. 
No obstante ante dudas de las constantes monitorizadas, y una vez que la situación de emergencia se supere, recomendamos una comprobación con otro manguito de presiones. terapéuticos a medio plazo

\section{BIBLIOGRAFÍA}

1. Farranco Ruiz, F et cols. Principios de urgencias, emergencias y cuidados críticos. Canulación Arterial. Disponible en: http://tratado.uninet.edu/c011808.html

2. Torné Perez, E. Canalización Arterial. Disponible en: http://www.enferpro.com/tarterial .htm

3. Llanos Ortega, MI. Inserción de catéteres arteriales. Disponible en: http://www.eccpn.aibarra.org/temario/seccion3/capitulo48/capitulo48.htm

4. Pickering TG, Hall JE, Appel LJ, Falkner BE, Graves J, Hill MN, et al. Recommendations for blood pressure measurements in humans and animals. Part 1: Blood pressure measurement in Humans: A statement for professional from Subcommittee of Professionals and Public Education on The American Heart Association Council on High Blood Pressure Research. Circulation. 2005;111:697-716.

5. Lapetra Peralta, J. et cols. Validación de monitores de presión arterial. Aten. Primaria. 16(5):295. 1995.

6. Agarwal R, Bunaye Z, Bekele D. Prognostic Significance of Between-Arm Blood Pressure Differences. Hipertensión. 2008; 51: 657-662.

7. Chobanian AV, Barkis GL, Black HR, Cushman WC, green LA, Izzo JL Jr,Jones DW, Materson BJ, oparil S, Wright TjJR, Rocella EJ. Seventh Report of the Joint National Committeé on Prevention, Detection, Evaluation, and Treatment of high Blood Pressure. Hypertension. 2003; 42: 1.206-1.252.

8. García, WJ. Et cols. Diferencia en la presión arterial entre ambos miembros superires. ¿Cuál es su verdadera magnitud?. Revista de posgrado de la Vía Cátedra de Medicina. № 125. Pág.: 32-35. Marzo 2003.

9. Araghi, A. et cols. Hipertensión arterial en la vigilancia en exceso de los pacientes críticos: invasiva o no invasiva?. Critical Care, 2006; 10(2).

10. Dopamina $\quad$ y norepinefrina. Disponible http://www.uam.es/departamentos/medicina/anesnet/agenda/farmacologia/dopamina.ht $\underline{\mathrm{m}}$ 